MATEC Web of Conferences 13, 05002 (2014)

DOI: $10.1051 /$ matecconf/ 20141305002

(C) Owned by the authors, published by EDP Sciences, 2014

\title{
Techno-Economic Assessment of Redundancy Systems for a Cogeneration Plant
}

\author{
Mohd Amin Abd Majid ${ }^{1, a}$ \\ Faculty of Mechanical Engineering, Universiti Teknologi PETRONAS
}

\begin{abstract}
The use of distributed power generation has advantage as well as disadvantage. One of the disadvantages is that the plant requires a dependable redundancy system to provide back up of power during failure of its power generation equipment. This paper presents a study on techno-economic assessment of redundancy systems for a cogeneration plant. Three redundancy systems were investigated; using public utility, generator set and gas turbine as back up during failures. Results from the analysis indicate that using public utility provides technical as well as economic advantages in comparison to using generator set or turbine as back up. However, the economic advantage of the public utility depends on the frequency of failures the plant will experience as well on the maximum demand charge. From the break even analysis of the understudied plant, if the number of failures exceeds 3 failures per year for the case of maximum demand charge of RM56.80, it is more economical to install a generator set as redundancy. The study will be useful for the co-generator operators to evaluate the feasibility of redundancy systems.
\end{abstract}

\section{Introduction}

Electrical power is an important commodity for public and industry. Normal practice is the electrical power is supplied by public utility (PU) company. In West Malaysia it is provided by Tenaga Nasional Berhad (TNB). In addition, there are also cases the power are provided by distributed power generators (DPG). The DPG have distinctly different size, fuel, efficiency and operating characteristics [1]. In many situations they provide considerable advantages with respect to the centaralised systems. Gas fueled co-generation (CG) plant falls under this category. To ensure reliable supply to consumers, the CG plants are normally provided with redundancy system to provide back up when GT failed. The normal practice is to fall back to PU for redundancy. This option leads to payment of expensive maximum demand (MD) charge. Other possible options are to use either generator set (GS) or gas turbine (GT) as redundancy. The choice of selecting the redundancy is normally left to the user. To avoid additional capital expenditure requirements, PU is normally chosen to provide redundancy, without considering the operation costs. From the experience of the CG operators, the use of PU can be expensive due to high cost of MD charge that is being imposed in using this facility. Studies related to issues on redundancy have been done, for example by $[2,3,4]$. Since there are many parameters involve due to various factors, such as capital and operating

\footnotetext{
${ }^{a}$ Corresponding author : mamin_amajid@petronas.com.my
}

This is an Open Access article distributed under the terms of the Creative Commons Attribution License 2.0, which permits unrestricted use, distribution, and reproduction in any medium, provided the original work is properly cited. 
expenditures and country specific regulations and practices, the findings on the published studies are not applicable locally. This study is to fcous on redundancy cost which relates to local CG plant.

\section{Methodology}

In this study three redundancy systems were considered namely; PU, GS and GT. Six technical criteria and two economic criteria have been identified as basis for comparison. The technical criteria are: capacity $(\mathrm{kW})$, fuel availability, modular, ease of switching, maintenance flexibility, reliability. The economic criteria are: capital and operation cost and maintenance cost. These are among the normal criteria which are used for assessment [1,5,6,7]. Pairwise comparison [5] and factor rating method [6] are adopted for technical assessment. For economic assessment net present value (NPV) method and break even analysis [7] were used.

\subsection{Technical Assessment}

The proposed technical assessment method was based on the evaluation approaches as proposed by [5] and [6]. The basis was to identify the criteria which influence technical performance. The weights of these criteria were evaluated using pairwise comparison. The factor rating method was used to evaluate the cumulative weighted scores of each alternative and select the preferred choice.

Reliability measurement of power could be based on frequency of interruption and duration [1]. To consider both factors would be difficult. System Average Interruption Duration Index (SAIDI) is used to measure reliability. SAIDI is the average of all interruptions, obtained by averaging over all of the utility customers [1]. TNB adopted SAIDI for reliability measurement of its power system [8,9]. In this study frequency of interruption was used as basis of reliability.

\subsection{Maximum Demand}

MD is the capacity of electricity usage, and it works to assess the level of capacity (load) of electricity used by customers. It is measured in kilowatts. The calculation of MD varies depending on the customer tariff categories and is only applicable to customers using a supply of $6.6 \mathrm{kV}$ and above [9]. MD charge depends on plant location and size. The charge is normally increases from time to time. For example for the case of CG plant at UTP, the MD charge increased from RM44.85 per kW in 2009 to RM48.60 in 2011 and now is RM56.80[10].

\subsection{Economic Assessment}

Annual cost (AC) was used as the basis of assessment. The choice of AC was to enable breakeven (BE) analysis to be done. $\mathrm{BE}$ analysis was done for the $\mathrm{AC}$ with respect to the frequency of failures (FoF) per year. Three cases were considered.

Case of using PU as redundancy: AC using PU was evaluated using Equation (1).

i. Case of operating co-generation

$\mathrm{C}_{\mathrm{pu}(\text { tot ann })}=\left[\mathrm{C}_{\mathrm{D}}+\mathrm{C}_{\mathrm{e}}+\mathrm{C}_{\mathrm{op}}\right] \times \mathrm{N}_{\mathrm{f}}$

ii. Case of co-generation under planning

$\mathrm{C}_{\mathrm{pu}(\mathrm{tot} \text { ann })}=\left[\mathrm{CR}_{\mathrm{ss}}+\mathrm{C}_{\mathrm{mss}}\right]+\left[\mathrm{C}_{\mathrm{D}}+\mathrm{C}_{\mathrm{e}}+\mathrm{C}_{\mathrm{op}}\right] \times \mathrm{N}_{\mathrm{f}}$

Case of GS as redundancy: Equation (3) was used to calculate the total AC.

$$
\mathrm{C}_{\text {gen (tot ann })}=\left[\mathrm{CR}_{\mathrm{g}}+\mathrm{C}_{\mathrm{mg}}\right]+\left[\left(\mathrm{C}_{\mathrm{og}}+\mathrm{C}_{\mathrm{op}}\right) \times \mathrm{N}_{\mathrm{f}}\right]
$$

Case of GT as redundancy: Equation (4) was used to determine the total AC.

where,

$$
\mathrm{C}_{\mathrm{t}(\mathrm{tot} \text { ann })}=\left[\mathrm{CR}_{\mathrm{t}}+\mathrm{C}_{\mathrm{mt}}\right]+\left[\left(\mathrm{C}_{\mathrm{ot}}+\mathrm{C}_{\mathrm{op}}\right) \times \mathrm{N}_{\mathrm{f}}\right]
$$

CRss $=$ capital recovery for substation $=\mathrm{Iss}(\mathrm{A} / \mathrm{P}, \mathrm{i}, \mathrm{Nss})-\mathrm{Sss}(\mathrm{A} / \mathrm{F}, \mathrm{I}, \mathrm{Nss})$

$\mathrm{CR}_{\mathrm{g}}=$ capital recovery for $\mathrm{GS}=\operatorname{Igen}\left(\mathrm{A} / \mathrm{P}, \mathrm{i}, \mathrm{N}_{\mathrm{g}}\right)-\mathrm{S}_{\mathrm{g}}\left(\mathrm{A} / \mathrm{F}, \mathrm{i}, \mathrm{N}_{\mathrm{g}}\right)$

$\mathrm{CR}_{\mathrm{t}}=$ capital recovery for $\mathrm{GT}=\mathrm{I}_{\mathrm{t}}\left(\mathrm{A} / \mathrm{P}, \mathrm{i}, \mathrm{N}_{\mathrm{t}}\right)-\mathrm{S}_{\mathrm{t}}\left(\mathrm{A} / \mathrm{F}, \mathrm{I}, \mathrm{N}_{\mathrm{t}}\right)$ 
$\mathrm{C}_{\mathrm{D}}=$ maximum demand charge per hook up, $\mathrm{C}_{\mathrm{e}}=$ cost of electricity per failure

$\mathrm{C}_{\mathrm{op}}=$ cost of opportunity loss per failure, $\mathrm{N}_{\mathrm{f}}=$ FoF per year

$\mathrm{C}_{\mathrm{og}}, \mathrm{C}_{\mathrm{ot}}=$ operation cost for GS and GT per failure respectively

$\mathrm{N}_{\mathrm{g}}, \mathrm{N}_{\mathrm{t}}=$ age of GS and GT respectively

$\mathrm{C}_{\mathrm{pu}}$ (tot ann), $\mathrm{C}_{\mathrm{g}}$ (tot ann) and $\mathrm{C}_{\mathrm{t}}$ (tot ann)= total AC using PU, GS and GT respectively

$\mathrm{C}_{\mathrm{mss}}, \mathrm{C}_{\mathrm{mg}}, \mathrm{C}_{\mathrm{mt}}=$ maintenance cost of substation, $\mathrm{GS}$ and $\mathrm{GT}$ per year respectively

$\mathrm{S}_{\mathrm{ss}}, \mathrm{S}_{\mathrm{g}}, \mathrm{S}_{\mathrm{mt}}=$ salvage value of substation, GS and GT respectively

\section{Results and discussion}

The proposed approach was used to assess three redundancy systems for a CG plant. The plant produced electricity and chilled water. The plant has two GT @ 4.2 MW each. The two GT were operated for 16 hours and the remaining 8 hours only one GT was operated. The average hourly kW was $7,600 \mathrm{~kW}$. During analysis it was assumed that when GT failed, power capacity equivalent to one GT only was taken from PU. It was also assumed that each failure was for one hour.

\subsection{Technical Assessment}

Three redundancy systems were considered; using PU, GS or GT, when the CG plant failed. In this study six technical criteria had been identified; capacity, fuel availability, modular, ease of switching to the backup, maintenance flexibility and reliability. These criteria are among the normal criteria which are used for technical assessment $[1,5]$. Results on the technical assessment are included in Table 1. The allocation of weights, were based on pairwise comparison method. Awarding of scores to each alternative was based on scales of 3,2 and 1, with 3 as the highest score and 1 as the least score.

Table 1. Technical Assessment of the Three Redundancy systems; PU, GS and GT

\begin{tabular}{|l|l|c|c|c|c|c|c|c|}
\hline No & Criteria & Weight & \multicolumn{2}{|c|}{ Public Utility } & \multicolumn{2}{c|}{ Generator Set } & \multicolumn{2}{c|}{ Turbine } \\
\cline { 4 - 9 } & & & Score & $\begin{array}{l}\text { Weighted } \\
\text { Score }\end{array}$ & Score & $\begin{array}{l}\text { Weighted } \\
\text { Score }\end{array}$ & Score & $\begin{array}{l}\text { Weighted } \\
\text { Score }\end{array}$ \\
\hline 1. & Capacity, kW & 0.133 & 3 & 0.399 & 1 & 0.133 & 2 & 0.266 \\
2. & Fuel availability & 0.333 & 3 & 0.399 & 2 & 0.666 & 1 & 0.333 \\
3. & Modular & 0.067 & 3 & 0.201 & 1 & 0.067 & 1 & 0.067 \\
4. & Ease of switching & 0.267 & 3 & 0.801 & 2 & 0.534 & 2 & 0.534 \\
5. & Maintenance flexibility & 0.067 & 3 & 0.201 & 2 & 0.134 & 2 & 0.134 \\
6. & Reliability & 0.133 & 3 & 0.399 & 2 & 0.266 & 2 & 0.266 \\
\hline & & & & 2.4 & & 1.8 & & \\
\hline
\end{tabular}

Capacity was considered as the most important criteria followed by fuel availability, modular, ease of switching, maintenance flexibility and lastly reliability. The basis of allocation the weight was that the selected redundancy system should be able to meet the capacity requirement. All the three systems were capable of meeting capacity requirements. However in terms of score PU scored 3 as it is the most capable to meet the capacity requirements followed by GT and GS.

Fuel availability is also important to drive the system and hence it is justified to be second in importance. Modular, ease of switching and maintenance ranking are appropriate. Since the redundancy system is to function for one hour. Reliability is ranked at last placing is acceptable.

The result on technical assessment arrives at PU as redundancy scored the highest weighted score. GS is second and GT is the third. Hence PU is the best redundancy for back up.

\subsection{Economic Assessment}

For the economic assessment, AC for the three alternatives namely PU, GS and GT, were calculated. Equations (1), (3) and (4), were used to calculate AC. The basis used were, service life of 15 years, interests rate of $10 \%$, MD of RM44.85 per kW, RM48.60 per kW and RM56.80 per kW. The MD of 
RM44.85 per kW was imposed from January 2009 until June 2011. From June 2011 until January 2014 the MD was RM48.60. From January 2014 until now the MD is 56.80 [10]. The rates per kWh were RM0.229 from January 2009 until June 2011, RM0.248 from June 2011 until January 2014. From January 2014 onwards the rate is RM0.290. The AC were evaluated with respect to the number FoF. The plots of total AC for the three alternatives versus FoF per year for MD RM44.85, RM 48.60 and RM56.80 are shown in Fig. 1, Fig 2 and Fig 3 respectively. The opportunity costs were excluded in the analysis for all the three cases as the values were similar. The plots indicate that MD influences the breakeven points of AC with respect to FoF. For the case MD is RM44.85 the breakeven point is at FoF of 3 (Figure 1). When MD is RM48.60 the breakeven point is at the point of slightly less than 3 (Figure 2). At MD RM56.80 the breakeven point is at the point FoF is 2 (Figure 5). Similar case when MD is RM60.00 (Figure 4). When MD was increased to RM65.00, the breakeven point for FoF is 2 . When the service life was reduced to 10 years there is minimal improvement to FoF to marginally higher than 2 (Figure 6). In summary, results of the analysis indicate an economic advantage of using PU as stand by for FoF up to 3 times a year. If FoF is greater than 3, the GS is more economic to be used as stand by. Even when the service life of GS was reduced to 10 years(Figure 5), the use of PU will not be economical comapared to GS when the FoF is 4 and greater.

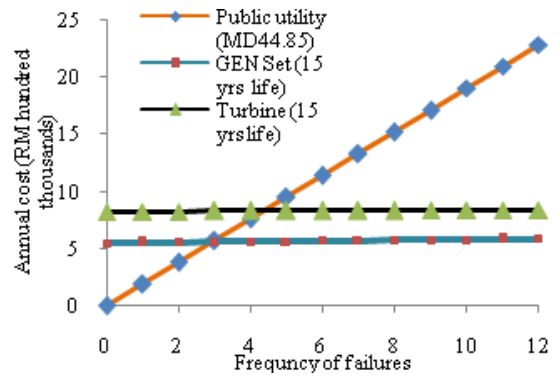

Figure 1. AC vs FoF for PU, GS and GT for MD RM44.85per $\mathrm{kW}$ and 4.2MW capacity based on 15 years service life.

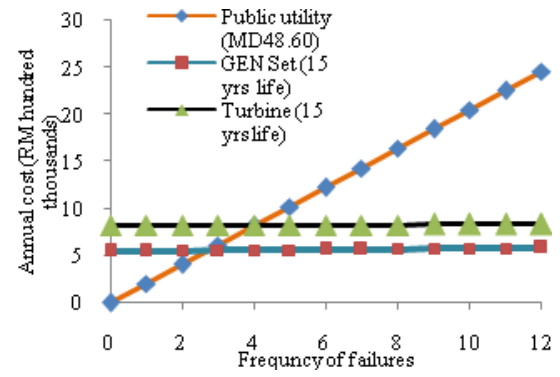

Figure 2. AC vs FoF for PU, GS and GT for MD RM48.60per $\mathrm{kW}$ and 4.2MW capacity based on 15 years service life.

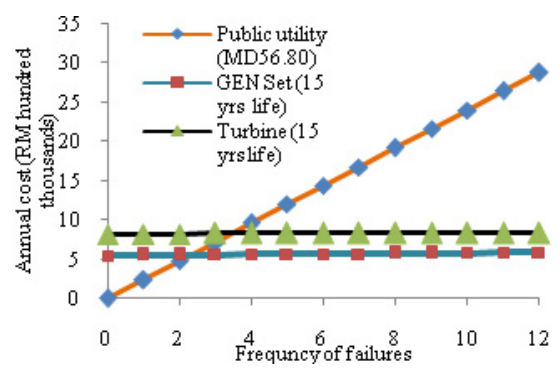

Figure3. AC vs FoF for PU, GS and GT for MD RM56.80 per $\mathrm{kW}$ and $4.2 \mathrm{MW}$ capacity based on 15 years service life.

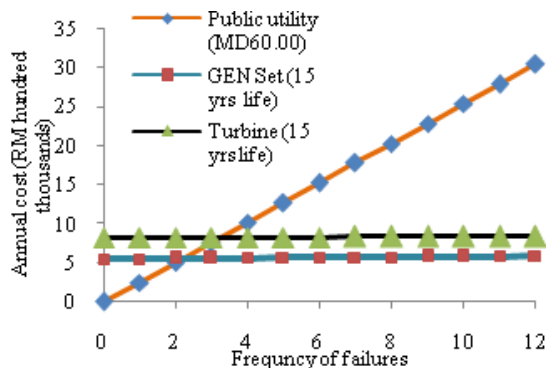

Figure 4. AC vs FoF for PU, GS and GT for MD RM60.00per $\mathrm{kW}$ and 4.2MW capacity based on 15 years service life.

Based on the analysis, the use of PU as standby has limited advantage. The constaraint is on FoF. The FoF sould not exceed 3 per year. Another contraint is that historical record indicates that MD shows an increasing trend. As highlighted above MD increased from RM44.85 to RM56.80 within a span of two and half years, from June 2011 to January 2014. An increase of about 27 per cent.

From the technical assessment, the PU option is found to be superior to GS or GT as redundancy. However from the economic evaluation the advantage of using PU as redundancy is only applicable for low number of FoF per year, for example for the understudied plant it is only up to 3 FoF. Once the FoF is higher than 3, it is more justified to switch to GS as redundancy. Hence the choice of redundancy system is very much dependence on economic advantage. 


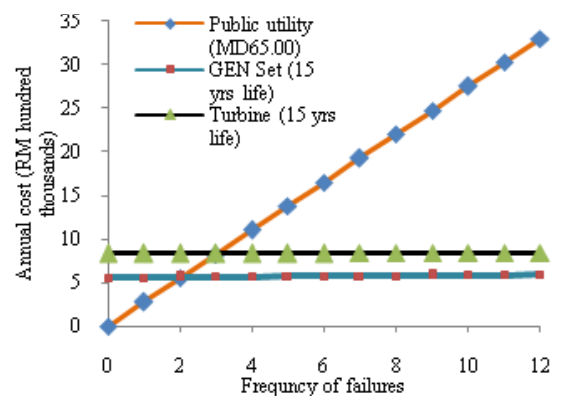

Figure5. AC vs FoF for PU, GS and GT for MD RM65.00per $\mathrm{kW}$ and 4.2MW capacity based on 15 years service life.

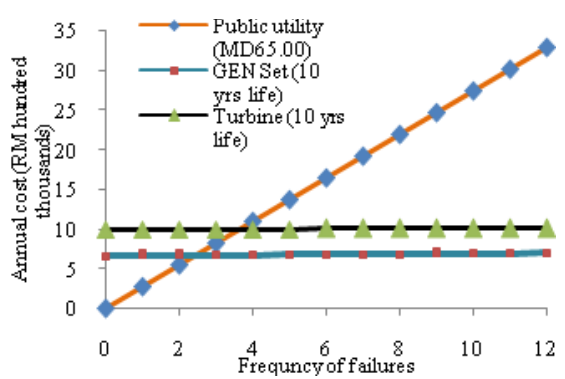

Figure 6. AC vs FoF for PU, GS and GT for MD RM65.00per $\mathrm{kW}$ and 4.2MW capacity based on 10 years service life.

\section{Conclusions}

In conclusion, using PU as redundancy provides technical and economic advantages in comparison to using GS or GT. However, this advantage depends on the rate of MD charge and the FoF per year. Increase of MD charge will lead to higher $\mathrm{AC}$ and the $\mathrm{PU}$ option may not be feasible. For case study CG plant, economic advantage of using PU is limited to FoF per year of 3 or less. When FoF is greater than 3 it is no more economically feasible. The plant should then switch to using GS for redundancy. Hence justification of selection of redundancy system, economic evaluation is important. For future work the study should consider to include the capacity required for redundancy other than 4.2MW.

\section{Acknowledgement}

Author would like to thank Universiti Teknologi PETRONAS and MOSTI for supporting this research study.

\section{References}

1. Willis, H. L. and Scott, W. G., Distributed Power Generation, Planning and Evaluation. Marcel Dekker, Inc., New York, (2000).

2. Kooten G. C., and Wong L., Economics of wind power when national grids are unavailable. Energy Policy 38:p. 1991-1998(2010).

3. Bridgwater A. V., Toft A. J.and Brammer J. G., A techno-economic comparison of power production by biomass fast pyrolysis with gasification and combustion. Renewable \& Sustainable Energy Reviews 6:p. 181-248(2002).

4. Steer S.J, Nuttall W.J., Parks J.T., and Goncalves L.V.N, Predicting the contractual cost of unplanned shutdowns of power stations: An accelerator-driven sub critical reactor case study. Electric Power Systems Research 81: p. 1662-1671 (2011).

5. Dieter G. E, and Schmidt L. C. Engineering Design, 5th Edition, Int. Edition. McGraw Hill, Singapore, (2013).

6. Heizer J., and Render, B. Operations management. 10th Edition. Pearson Higher , (2010).

7. Sullivan, Wicks, Koelling. Engineering Economy. 15th Edition, International Edition, Pearson Education Ltd., (2012).

8. Farik Zolkepli, TNB targets improving on power outage index. NATION, (Sat. June 1, 2013)

9. TNB, Maximum Demand, http://www.tnb.com.my/business/for-commercial/maximumdemand.html, retrieved on (15th Jan 2014).

10. Suhala S., Customised Contract Hume, Cogen UTP, TIK General-Medium Voltage Peak/Off Peak Commercial Bulk Supply Tariff. e-mail from Suhala Sukarno to Azanin Bismi A., Cheah P.W, (15th January 2014). 